\title{
Administração de medicamentos: orientação final de enfermagem para a alta hospitalar*
}

\author{
ADMINISTRATION OF MEDICINES: FINAL NURSING ORIENTATION FOR HOSPITAL RELEASE \\ ADMINISTRACIÓN DE MEDICAMENTOS: ORIENTACIÓN FINAL DE ENFERMERÍA \\ PARA EL ALTA HOSPITALARIA
}

\section{Adriana Inocenti Miasso', Silvia Helena De Bortoli Cassiani²}

\footnotetext{
Extraído da Dissertação "Terapêutica medicamentosa: orientação e conhecimento do paciente na alta e pós-alta hospitalar", Escola de Enfermagem de Ribeirão Preto - Universidade de São Paulo (EERP-USP), 2002.

1 Enfermeira. Doutoranda do Programa Interunidades de Doutoramento em Enfermagem da EERP-USP. amiasso@zipmail.com.br.

3 Professora Associada do Departamento de Enfermagem Geral e Especializada da EERP-USP
}

\begin{abstract}
RESUMO
Este estudo avaliou a orientação final de enfermagem para a alta hospitalar quanto à terapêutica medicamentosa em uma clínica de internação hospitalar. A amostra constou de 38 pacientes com alta hospitalar em dezembro de 2001 e fevereiro de 2002 e dos enfermeiros que os orientaram. Para coleta dos dados utilizou-se a técnica de observação não participante. Obtivemos como resultados: locais inadequados para orientação, poucas informações por escrito, curto tempo para orientação e não utilização de estratégias que confirmem o entendimento do paciente quanto as orientações. Conclui-se que estes aspectos possam estar contribuindo para ocorrência de erros de medicação no domicílio, após a alta hospitalar.
\end{abstract}

\section{DESCRITORES}

Erros de medicação.

Alta do paciente.

Educação do paciente.

\section{ABSTRACT}

This study assessed the final nursing orientation for hospital release in what refers to the medicamental therapeutics in a clinic of hospital internment. The sample was comprised of 38 patients that had hospital release in December of 2001, February of 2002 and of the nurses who oriented them. The technique used for collecting data was nonparticipant observation. The results obtained were: inadequate places for orientation, few written information, short time for orientation and no use of strategies that confirm the comprehension of the orientation on the part of the patient. The conclusion is that those problems may be contributing for the occurrence of errors in the use of medicines at home, after hospital release.

\section{KEY WORDS}

Medication errors.

Patient discharge.

Patient education.

\section{RESUMEN}

Este estudio evaluó la orientación final de enfermería para el alta hospitalaria en cuanto a la terapéutica medicamentosa en una clínica de internamiento hospitalario. La muestra constó de 38 pacientes con alta hospitalaria en diciembre del 2001, febrero del 2002 y de los enfermeros que los orientaron. Para la recolección de los datos se utilizó la técnica de observación no-participante. Obtuvimos como resultados: locales inadecuados para la orientación, pocas informaciones por escrito, corto tiempo para la orientación y no utilización de estrategias que confirmen el entendimiento del paciente en cuanto a las orientaciones. Se concluyó que tales aspectos pueden estar contribuyendo para la ocurrencia de errores de medicación en el domicilio, después del alta hospitalaria.

\section{DESCRIPTORES}

Errores de medicación.

Alta del paciente.

Educación del paciente. 


\section{INTRODUÇÃO}

Erros relacionados à administração de medicamentos podem ocorrer tanto no hospital quanto no domicílio, após a alta hospitalar do paciente. Sabe-se que muitas vezes o paciente recebe alta hospitalar com a prescrição de um grande número de medicamentos necessários à continuidade do seu tratamento e em decorrência de vários fatores acaba ocorrendo erros.

A ocorrência de erros de medicação no domicílio tem relação com o trabalho do enfermeiro, uma vez que é de competência desse profissional educar o paciente para o uso correto dos medicamentos após a alta hospitalar.

Para tanto o enfermeiro precisa ter conhecimento da ação do medicamento no organismo, métodos e vias de administração, eliminação, reações colaterais, dose máxima e terapêutica, efeitos tóxi$\cos$, das técnicas de administração pelas diferentes vias, bem como, da anatomia e fisiologia humana pois, falhas no conhecimento do enfermeiro refletem diretamente na orientação que é fornecida ao paciente.

Estudo detectou conhecimento deficiente dos enfermeiros sobre o nome comercial e genérico do medicamento, propriedades farmacológicas, cálculo errado do fluxo de infusão intravascular, conhecimento insuficiente acerca das indicações do medicamento e falta de preparo teórico para subsidiar a implementação segura da terapia medicamentosa $^{(1)}$.

Aliado a este contexto observa-se atualmente que, devido à carência de recursos nas instituições hospitalares, há uma tendência do retorno mais precoce do paciente ao domicílio, diminuindo o período disponível para educação do mesmo ${ }^{(2)}$.

O "plano de alta", como garantia da continuidade da assistência do paciente após sua hospitalização, pode ser uma saída e deve-se considerar a importância do envolvimento da família em todas as etapas do plano, devendo para isso, ser orientada e compreender o estado de saúde e necessidades do paciente ${ }^{(3)}$.

O enfermeiro deve avaliar as habilidades do paciente para se auto-cuidar e o interesse da família em ajudá-lo, visto que o plano de alta tem como finalidade tornar o paciente auto-suficiente para seu cuidado no domicílio ou para ser cuidado pela família. Desse modo, o papel do enfermeiro no processo de alta é o de proporcionar assistência desde a internação, educando o paciente e a família.
O profissional e o paciente devem constantemente avaliar seu progresso no que se refere ao processo ensino-aprendizagem, uma vez que a mudança de comportamento do paciente constitui um importante fator para a garantia da continuidade de sua assistência no domicílio(4).

Quanto às orientações finais estabelecidas no plano de alta, o paciente deve recebê-las antes do horário previsto para sua saída formal do hospital, evitando o acúmulo de informações nesse momento, possibilitando a avaliação de sua compreensão quanto às informações fornecidas e o esclarecimento de dúvidas.

Assim sendo, no momento da alta, o enfermeiro deve reforçar as orientações sobre o plano a ser seguido e a importância do retorno para controle médico (caso seja solicitado pelo médico). O paciente deve ser informado que poderá se comunicar com a equipe sempre que tiver alguma dúvida. Um telefone que possa ser fornecido para contato com a equipe hospitalar é sempre uma alternativa importante no caso de dúvidas.

Grande parte dos pacientes deixa o hospital com dúvidas sobre os medicamentos prescritos, dieta a ser seguida, retornos para seguimento e das atividades que poderá realizar e há ainda um número reduzido de informações redigidas pela enfermagem e fornecidas aos pacientes. $\mathrm{O}$ conhecimento insuficiente e a carência de entendimento podem ocasionar uma administração de medicamentos incorreta e ineficiente ${ }^{(5-6)}$.

Reconhecemos que os pacientes devem ter conhecimento de todos os aspectos de seu cuidado, incluindo a terapia medicamentosa. Devem também assumir papel ativo no uso de seus medicamentos através do questionamento e aprendizagem de seu tratamento pois, assim, a ansiedade relacionada à incerteza do tratamento pode ser aliviada e erros podem ser prevenidos.

\section{OBJETIVO}

Avaliar a orientação final de enfermagem para a alta hospitalar no que concerne à terapêutica medicamentosa, em uma clínica de internação de um hospital universitário do interior paulista.

\section{METODOLOGIA}

\section{Tipo de estudo}

Trata-se de um estudo descritivo, transversal e de campo.
Administração de medicamentos: orientação final de enfermagem para a alta hospitalar 
Adriana Inocenti Miasso Silvia Helena De B. Cassiani

\section{População e amostra}

A população alvo deste estudo compõe-se de todos os pacientes e enfermeiros da clínica médica de um hospital universitário do interior do estado de São Paulo.

A amostra investigada constou de 38 pacientes adultos, de ambos os sexos, que receberam alta hospitalar, com prescrição de medicamentos para uso no domicílio, nos meses de dezembro de 2001 e fevereiro de 2002 e dos enfermeiros responsáveis pela orientação final para alta hospitalar desses pacientes.

\section{Coleta de dados}

Os dados foram coletados após a aprovação do projeto pelo Comitê de Ética de Pesquisa em Seres Humanos da instituição hospitalar e conforme Resolução 196/96.

Para a coleta dos dados, a autora permaneceu na clínica em estudo, diariamente, a partir do horário em que os médicos residentes chegam à clínica ( 8 horas), até o término da observação da orientação de enfermagem ao último paciente de alta hospitalar do dia (nos meses de dezembro de 2001 e fevereiro de 2002). A confirmação da última alta hospitalar do dia era obtida junto aos enfermeiros e médicos residentes da unidade.

No momento da alta, era realizada a observação direta não participante da orientação de enfermagem ao paciente, com a utilização de um roteiro que continha dados referentes ao ambiente, prescrição médica, orientação de enfermagem sobre a terapêutica medicamentosa e dados relativos ao paciente e família. Os dados a serem observados não foram revelados antecipadamente aos profissionais da unidade com a finalidade de evitar interferências no comportamento dos mesmos. Os dados oriundos de cada observação eram registrados no roteiro após o término da mesma. Foi solicitado o consentimento informado aos participantes do estudo.

\section{Análise dos dados}

Foi realizada análise quali-quantitativa dos dados. A partir das notas redigidas foram extraídas as seguintes unidades de análise: o ambiente da orientação e a prescrição médica, o conteúdo da orientação, o paciente e a família. Nessa etapa foram criados alguns enunciados a partir das respostas dos pacientes. Tais enunciados se limitaram a conter as sentenças chaves extraídas das respostas.

\section{RESULTADOS E DISCUSSÃO}

No aprendizado do paciente, as dificuldades que surgem podem estar relacionadas ao orientador, ao aprendiz (quando este demonstra pouco interesse e disponibilidade, tem baixa motivação e capacidade de aprendizagem, entre outras), ou ao ambiente onde se desenvolve a situação. A falta de apoio dos familiares também pode interferir no aprendizado do paciente ${ }^{(7)}$.

Assim, os resultados desse estudo serão apresentados sob quatro enfoques: o ambiente da orientação e a prescrição médica, o conteúdo da orientação, o paciente e a família.

\section{O Ambiente da orientação e a prescrição médica}

\section{Ambiente}

No que se refere ao ambiente utilizado para a orientação final de enfermagem ao paciente de alta hospitalar, verificou-se que para $18(47,4 \%)$ participantes a orientação foi realizada com o enfermeiro dentro do posto de enfermagem e o participante em frente ao posto, $7(18,4 \%)$ participantes foram orientados dentro da enfermaria, para $6(15,8 \%)$ participantes a orientação foi realizada com o enfermeiro e o participante em frente ao posto de enfermagem e $4(10,5 \%)$ participantes foram orientados no corredor interno próximo às enfermarias. Identificou-se, ainda, 1 (2,6\%) participante que foi orientado em frente ao elevador por um auxiliar de enfermagem, 1 (2,6\%) que foi orientado em frente ao posto de enfermagem por um auxiliar de enfermagem e $1(2,6 \%)$ participante que recebeu orientação do enfermeiro no refeitório.

Destaca-se o fato de que $65,8 \%$ dos participantes foram orientados próximo ao posto de enfermagem, ambiente este considerado inadequado para esta atividade tendo em vista o grande fluxo de pessoas, a entrega e recolhimento de materiais, o excesso de ruídos referente a rádio ligado, telefone tocando, pessoas conversando, dentre outros fatores presentes neste ambiente que podem contribuir para o desvio de atenção do paciente e enfermeiro durante a orientação.

Ainda com relação à caracterização do ambiente utilizado para orientação ao participante, foi possível verificar que $1(2,6 \%)$ participante foi orientado com a presença de rádio ligado e 3 $(7,9 \%)$ com a televisão ligada. Durante a orientação de 15 participantes, o telefone tocou. Todas as orientações de enfermagem foram realizadas em ambientes com pessoas transitando e con- 
versando o que poderia dificultar a audição acerca da orientação.

Descrevemos os vários fatores que podem contribuir para o desvio de atenção do enfermeiro/participante no momento da orientação tais como ruídos, fluxo de pessoas entre outros. Nesse momento consideraremos desvio de atenção do enfermeiro/participante a interrupção da orientação de enfermagem.

A esse respeito obtivemos que: 3 enfermeiros foram abordados pelo médico e interromperam a orientação para fornecer ao mesmo informações sobre outro paciente, 3 enfermeiros pararam a orientação para atender o telefone, 1 foi abordado pelo escriturário e o atendeu e 1 enfermeiro foi abordado por uma funcionária que o comunicou que a medicação das oito horas não havia sido administrada ao participante receptor da orientação e, em seguida o enfermeiro preparou e administrou a medicação. Cabe informar que tal orientação foi realizada às dez horas e quinze minutos e, desse modo, a medicação foi administrada com duas horas e quinze minutos de atraso.

Acreditamos que a interrupção da orientação ao paciente ocasionou perda de informações importantes à continuidade de seu tratamento bem como, diminuiu sua liberdade para expressar-se e questionar.

Desse modo, pode-se considerar que todos os locais utilizados para orientação ao paciente foram inadequados. Para realização desta atividade deveria ser utilizado um local reservado, com ausência de ruídos, onde o enfermeiro pudesse realizar a orientação ao paciente e familiar sem que ocorresse interrupções e desvio de atenção dos mesmos.

As dificuldades de aprendizagem do participante podem situar-se nos estímulos excessivos do local onde ocorre a orientação bem como na falta de local apropriado (ruídos, temperatura, iluminação e privacidade) para realização das mesmas ${ }^{(7)}$.

\section{Prescrição médica}

Observando as receitas médicas destinadas aos participantes, identificamos que todas continham a dosagem dos medicamentos, a freqüência de utilização dos mesmos bem como o uso de abreviaturas. Verificamos ainda que 9 receitas apresentavam letra parcialmente legível, o que dificultava a identificação do nome e dosagem de alguns medicamentos, em 4 não constava o tempo de utilização dos medicamentos, em 3 receitas todos os medicamentos foram prescritos pelo nome comercial e em 11 havia no mínimo um medicamento prescrito pelo nome comercial.
Sabe-se que a receita médica constitui uma fonte de informações a qual o paciente pode recorrer caso tenha dúvidas sobre a utilização de seus medicamentos. Desse modo os médicos deveriam dispensar atenção especial à elaboração da prescrição dos medicamentos para uso após a alta hospitalar, visando à prevenção de erros de medicação no domicílio.

A orientação inadequada ao participante sobre seus medicamentos durante a internação e o fornecimento de uma receita médica parcialmente legível e/ou com informações ambíguas no momento da alta hospitalar constituem fatores potenciais para os erros de medicação no domicílio.

Passaremos agora à análise do conteúdo da orientação de enfermagem aos participantes do estudo que estavam de alta hospitalar.

\section{O Conteúdo da orientação}

O enfermeiro, por atuar diretamente na administração de medicamentos ao paciente, tem papel primordial na orientação do mesmo, no que se refere à terapêutica medicamentosa.

Nesse estudo verificamos que dos 38 participantes, $36(94,7 \%)$ foram orientados por enfermeiros e $2(5.3 \%)$ por auxiliares de enfermagem. Estes dois últimos participantes recusaram-se a aguardar a orientação do enfermeiro para alta alegando pressa em deixar o hospital. Um dos participantes estava deixando a clínica sem receber as orientações finais, sendo abordado e orientado pelo auxiliar de enfermagem em frente ao elevador. Vale ressaltar que de acordo com o manual de normas e rotinas da instituição em estudo, constitui função do enfermeiro a orientação de pacientes de alta hospitalar.

Quanto ao tempo de duração da orientação final de enfermagem ao paciente de alta hospitalar, constatamos que $28(73,7 \%)$ orientações tiveram duração de 2 a 5 minutos, $7(18,4 \%)$ duraram de 6 a 10 minutos e 3 (7,9\%) orientações tiveram duração superior a 10 minutos.

Cabe ressaltar que o tempo de orientação mencionado foi utilizado para fornecer informações gerais ao paciente incluindo àquelas da terapêutica medicamentosa prescrita.

Não encontramos na literatura estudos que estabelecessem um tempo adequado para orientação do paciente de alta hospitalar uma vez que o tempo utilizado depende de uma série de fatores inerentes ao paciente e tratamento estabelecido. Entretanto, partindo-se de nossa experiência e do
Administração de medicamentos: orientação final de enfermagem para a alta hospitalar 
Adriana Inocenti Miasso Silvia Helena De B. Cassiani pressuposto de que o paciente tenha sido orientado durante a internação quanto aos aspectos relevantes para a continuidade de seu tratamento, consideramos que um tempo de orientação inferior a 10 minutos seria insuficiente para reforçar as orientações referentes ao uso de medicamentos, exames, retornos, cuidados específicos tais como alimentação, atividade física, entre outros. Cabe mencionar que para $92,1 \%$ dos participantes a orientação final de enfermagem teve duração igual ou inferior a 10 minutos.

No que se refere à orientação de enfermagem por escrito ao participante, para $3(7,9 \%)$ participantes foram anotados, na receita médica, os horários em que deveriam tomar os medicamentos de acordo com os horários em que os utilizavam no hospital.

Na educação do paciente, tanto a informação verbal quanto a escrita são importantes e complementares. A informação verbal é insuficiente devido ao fato de o paciente priorizar as informações relativas ao diagnóstico em detrimento das informações sobre o medicamento. Tem-se ainda a possibilidade de o paciente não compreender a informação verbal, esquecê-la ou rejeitá-la. O fornecimento de informações escritas, através de folhetos explicativos, tem sido uma maneira efetiva de apoiar as orientações verbais fornecidas ao paciente a respeito dos medicamentos ${ }^{(8)}$.

Com relação às informações fornecidas ao participante sobre o(s) medicamento(s) prescritos para uso no domicílio, verificamos que 25 enfermeiros leram a receita médica para o participante, 2 apenas entregaram a receita, 6 perguntaram ao participante se o médico havia entregue a receita e não orientou o participante sobre os medicamentos, 3 enfermeiros entregaram a receita e disseram: "Continua as mesmas medicações". Os dois auxiliares de enfermagem leram a receita médica para o participante.

Verifica-se que a maioria dos enfermeiros $(65,8 \%)$ limitou-se a ler a receita médica para o participante e, desse modo, forneceu aos mesmos apenas as informações nela contidas, as quais, como já descrito, eram incompletas.

Atualmente, na rotina da alta hospitalar, observa-se que a responsabilidade da equipe de enfermagem acaba no momento em que os papéis estão sendo preenchidos e a receita médica está em posse do paciente ou de seus familiares, o que também pode ser comprovado nesse estudo $^{(9)}$.
Para que o paciente reconheça a necessidade dos medicamentos para manutenção de sua saúde e tenha uma participação ativa no tratamento de sua doença, é necessário que receba uma orientação adequada. Essa orientação deve incluir entre outros aspectos, a finalidade dos medicamentos, o modo de utilização, os cuidados que devem ser tomados com os mesmos e como lidar com possíveis efeitos colaterais. Deve ainda enfatizar a importância da continuidade do tratamento.

Antes de realizar as orientações, o enfermeiro deve verificar se o paciente sabe ler, com o objetivo de derivar estratégias que facilitem o aprendizado do mesmo. Vale ressaltar que nenhum enfermeiro ou auxiliar de enfermagem que atuou na orientação ao participante identificou se o mesmo sabia ler.

Com relação às estratégias utilizadas para verificar se o participante compreendeu as orientações fornecidas, obtivemos que 33 (86,8\%) enfermeiros não verificaram se o participante compreendeu as orientações e $5(13,2 \%)$ enfermeiros, após ler a receita médica, perguntaram aos participantes se os mesmos haviam entendido as orientações.

A maioria dos enfermeiros não assume as responsabilidades em relação às orientações ministradas, gerando a idéia de que uma vez realizada a orientação, o profissional já cumpriu seu papel, sem preocupar-se se o paciente assimilou ou não as informações fornecidas ${ }^{(7)}$.

O enfermeiro deve assegurar-se de que o paciente compreendeu as orientações ministradas e, pedir para que o paciente as repita constitui uma medida eficaz para o alcance desse objetivo.

Durante a internação, o participante deve receber todas as informações necessárias ao uso correto de seus medicamentos. Deve ainda ser encorajado a exercer papel ativo no seu tratamento e questionar sempre que houver dúvidas.

\section{Enfocando o paciente}

Ao orientar o paciente o enfermeiro deve considerar entre vários aspectos: as expectativas do mesmo quanto à alta hospitalar, o conhecimento prévio sobre sua doença e tratamento, sua cultura e condições sócio-econômicas.

A grande quantidade e diversidade de experiências e conceitos adquiridos previamente e a atividade educacional podem influenciar positivamente ou não na sua nova aprendizagem( ${ }^{(7)}$. 
Durante a observação da orientação terapêutica de enfermagem, no que se refere ao paciente, vários aspectos foram observados e serão discutidos a seguir. Pela riqueza das observações e falas dos pacientes, decidimos estar incluindo algumas das falas mais significativas para o estudo dessa temática, como forma de ilustrar o conteúdo discutido.

Durante a orientação na alta hospitalar, dos 38 participantes, $11(28,9 \%)$ apresentaram dúvidas quanto aos medicamentos prescritos para uso no domicílio. Destes, 3 questionaram sobre a dose do medicamento ${ }^{(\mathrm{a})}$.

Eu tenho que tomar 1 comprimido ou meio comprimido de clorana? (p.12)

Durante a internação este paciente fez uso de meio comprimido de clorana por dia e ao ser por nós questionado, antes da orientação final de enfermagem, se conhecia os medicamentos utilizados na internação, não mencionou o nome deste medicamento, o que demonstra que o participante não sabia que o estava utilizando durante a internação.

Foi também prescrito para este participante utilizar no domicílio: "Meticorten 5mg, tomar 1,5 cp cedo em dias alternados" e o mesmo questionou:

O meticorten não é de 20 mg? (p 33).

Observando a prescrição feita para o paciente no último dia de internação verificamos que foi prescrito para o mesmo: "Prednisona 20mg, 1,5 cp cedo em dias alternados". O enfermeiro orientou o participante a telefonar para a clínica no dia seguinte com o objetivo de confirmar a dose do medicamento com o médico. Quando questionado no domicílio, o participante informou que houve um erro na prescrição e o médico o orientou, por telefone, a utilizar $30 \mathrm{mg}$ por dia do medicamento e a comparecer no hospital para realizar a troca da receita.

Essa situação denota a importância do conhecimento do paciente a respeito do medicamento que utiliza, já que lhe fornece subsídios para questionamentos bem como, para opinar sobre a terapêutica medicamentosa empregada. Nesse caso especificamente, evitou um erro de medicação no domicílio, assegurando a utilização da dose correta do medicamento.

Para outro participante foi prescrito: "LCarnitina 500mg/ml, tomar $2 \mathrm{ml} \mathrm{de} \mathrm{12/12h} \mathrm{(4g/dia)"}$ o qual indagou:
Em casa eu tenho frasco de $250 \mathrm{mg} / \mathrm{ml}$, então quantos $\mathrm{ml}$ eu tenho que tomar? (p.38).

O enfermeiro o orientou a tomar $4 \mathrm{ml} \mathrm{em} \mathrm{cada}$ horário. Fica evidente que o participante apresentou dificuldade em realizar um cálculo de fundamental importância para o uso correto da dose do medicamento prescrito. Além de pedir ao paciente que repita as informações fornecidas, o enfermeiro deve estimulá-lo a expressar suas dúvidas, perguntar-lhe se possui em casa algum dos medicamentos prescritos e qual a dosagem do mesmo, tendo em vista a variedade de formas de apresentação dos medicamentos e a dificuldade de alguns pacientes em realizar os cálculos necessários ao aproveitamento do medicamento que possui.

Também identificamos que 3 participantes questionaram sobre o nome do medicamento. Ao ler a receita para o participante "Amitriptilina $25 \mathrm{mg}$, to-

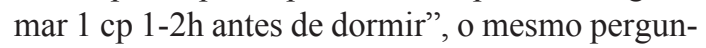
tou:

Essa medicação é nova? (p.1).

Ao verificarmos a prescrição feita para o participante durante a internação constatamos que o mesmo não estava utilizando este medicamento. Como o participante não foi informado de que faria uso do medicamento no domicílio, conseqüentemente não recebeu informações sobre o mesmo.

Cabe informar que o cloridrato de amitriptilina constitui um antidepressivo que pode ocasionar, no usuário, uma série de efeitos colaterais. Destaca-se entre os mais freqüentes: secura na boca, hipotensão, turvação visual, constipação, íleo paralítico, tontura, fraqueza, fadiga, aumento ou perda de peso, sonolência, impotência, entre outros. Além de fornecer as informações presentes na prescrição médica, caberia ao enfermeiro orientar o participante enfatizando quanto aos possíveis efeitos colaterais decorrentes da utilização do medicamento e os cuidados a serem tomados na presença dos mesmos, tendo em vista que a medicação seria iniciada no domicílio, sem uma monitorização próxima da equipe de saúde.

Foi prescrito para um participante utilizar no domicílio: "Insulina NPH 12U cedo e Insulina regular 4U cedo" e o mesmo indagou:

Tem dois tipos de insulina? (p10).

Durante a internação o participante utilizou Insulina de ação intermediária 16UI de manhã e 4UI à tarde. Assim, para uso no domicílio, ocorreram mudanças no que se refere ao tipo, dosagem e horário de administração do medicamento e o pacien-
Administração de medicamentos: orientação final de enfermagem para a alta hospitalar

\footnotetext{
(a) Algumas falas selecionadas foram transcritas para uma melhor compreensão dos problemas. Elas são acompanhadas de uma codificação entre parênteses, que relaciona a resposta ao indivíduo observado. Assim, o código (p.1) corresponde ao participante observado que recebeu o número de ordem 1.
}

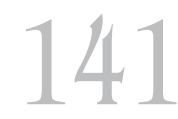

Rev Esc Enferm USP 2005; 39(2):136-44 
Adriana Inocenti Miasso Silvia Helena De B. Cassiani te só recebeu essa informação no momento da alta hospitalar.

Este paciente solicitou ainda que fosse escrito em sua receita a palavra "humana", pois em pós-alta anterior não conseguiu adquirir a insulina na Unidade Básica de Saúde devido a ausência dessa palavra na prescrição médica. $\mathrm{O}$ enfermeiro orientou o participante que no hospital o mesmo havia feito uso de insulina suína e não de insulina humana. Diante de tal informação, o paciente mostrou-se ansioso, pediu para conversar com o médico e questionou:

Então a insulina que eu tomei em casa tava errada e descompensou meu diabetes? (p.10).

O médico foi comunicado e realizou as orientações necessárias ao paciente.

Todas as informações referentes à insulina: tipos, horários e locais adequados à administração, entre outras deveriam ter sido fornecidas ao participante durante a internação visando prepará-lo para o uso correto do medicamento após a alta hospitalar.

No momento da alta o enfermeiro orientou ao participante que deveria utilizar "Tiroxina $75 \mathrm{mg}$, 1 comp. cedo" e o mesmo questionou:

Esse medicamento é novo?...Eu tomava Puran antes (p. 16)

O enfermeiro respondeu ao paciente: Agora é esse. A resposta emitida pelo enfermeiro denota o seu desconhecimento quanto ao medicamento prescrito, uma vez que ambos os nomes referem-se a um mesmo princípio ativo. Assim, o participante recebeu alta hospitalar com a informação de que faria uso de um medicamento que não utilizava anteriormente.

Verificamos que um participante questionou se poderia fazer uso de bebida alcoólica durante o uso do medicamento e o enfermeiro limitou-se a dizer é melhor não. Um questionou sobre o nome e indicação do medicamento (perguntou se no caso não encontrar o enalapril poderia comprar o eupressin e o enfermeiro respondeu que se tratava do mesmo medicamento). Perguntou ainda o que era ticlid e o enfermeiro respondeu é um anti-hipertensivo).

Identificamos um participante com dúvidas quanto ao nome e interação com alimentos. Questionou: esse medicamento é novo? referindo-se a penicilamina e se eu tomar os medicamentos com leite faz mal?. O enfermeiro respondeu respectivamente esse é novo e não, pode tomar com leite.
Temos um participante que questionou porque não poderia deitar-se após uso do medicamento prescrito e, nesse caso o enfermeiro solicitou ao médico que explicasse ao paciente. Um participante perguntou porque utilizou polaramine apenas um dia e o medicamento não foi prescrito para uso no domicílio e o enfermeiro disse que não sabia informar, que o participante deveria perguntar ao médico.

As situações descritas mostram que o enfermeiro, embora seja responsável pela administração de medicamentos bem como, pela orientação dos pacientes sobre os vários aspectos relativos à terapêutica medicamentosa, nem sempre possui uma base adequada de conhecimento para assumir essa responsabilidade.

Um estudo que investigou falha no conhecimento de profissionais de enfermagem a respeito da administração de medicamentos e suas propriedades farmacológicas, detectou conhecimento deficiente sobre o nome comercial e farmacológico do medicamento, suas indicações, propriedades farmacológicas, cálculo incorreto do fluxo de infusão intravascular e falta de preparo teórico para subsidiar a implementação segura da terapia medicamentosa ${ }^{(1)}$.

\section{Enfocando a família}

Sabe-se que cada vez mais a família tem assumido a responsabilidade de continuar cuidando da saúde de seus membros, necessitando, portanto, de apoio dos profissionais no que se refere à atenção à saúde, tanto no âmbito hospitalar quanto domiciliar. Em relação à administração de medicamentos, deve ser orientada tanto quanto o paciente para que possa auxiliá-lo nessa tarefa caso necessite.

Assim, consideramos de fundamental importância a presença da família durante as orientações ao paciente. A esse respeito, observamos que dos 38 participantes, 27 (71\%) estavam acompanhados por familiares durante a orientação final de enfermagem para alta hospitalar. Destes, 12 estavam acompanhados por esposo/esposa, 6 por filho/filha, 4 por pai/mãe, 3 por irmão/irmã e 2 participantes estavam acompanhados pela nora.

Verificamos que 3 familiares apresentaram dúvidas durante as orientações:

Como faz quando o paciente começa a melhorar e não quer mais tomar o remédio?

(p 2). 
Esse questionamento do familiar nos remete à hipótese de que em algum momento do tratamento o participante deixou de utilizar os medicamentos prescritos. Cabe então ao enfermeiro, diante do interesse do familiar por informações, expor ao mesmo e ao participante os resultados benéficos e importantes que o tratamento correto proporciona, assim como os adversos que comumente pode experimentar e o que o participante deveria fazer nesse último caso, estimulando-o a desenvolver a auto-responsabilidade pelo seu tratamento. $\mathrm{O}$ enfermeiro orientou o familiar e o paciente que o não seguimento da terapêutica medicamentosa poderia causar uma piora da doença.

Quando o nível de informação se amplia, o paciente torna-se mais eficiente no sentido de ter uma participação mais ativa no tratamento de sua doença e de se auto-cuidar. Uma orientação adequada estimula a motivação do paciente para tomar o medicamento corretamente visando o alcance da cura ou melhoria de sua condição de saúde ${ }^{(10)}$.

Quanto aos medicamentos que já utilizava, observamos a seguinte questão de 1 participante:

E os remédios que estava tomando? (p. 30)

O enfermeiro orientou que o participante deveria utilizar apenas os medicamentos que constavam na receita atual. A experiência mostra que alguns pacientes acreditam que devem suspender os medicamentos que já utilizavam, para evitar que interfiram com os recém-prescritos outros, após a alta, somam os medicamentos recém-prescritos aos que utilizavam anteriormente à internação sem questionar sobre a necessidade dos mesmos ou possíveis interações com os medicamentos atuais. Ressalta-se a importância de orientar o paciente a informar, por ocasião da admissão, o nome de todos os medicamentos que utiliza para que os profissionais da saúde possam orientá-lo sobre possíveis interações com os medicamentos recém-prescritos, necessidade de suspender algum medicamento devido à introdução de outro, bem como para orientá-los sobre quais medicamentos deverá utilizar após a alta hospitalar. Entretanto não basta que o paciente informe o nome dos medicamentos, é necessário que os profissionais responsáveis pelo paciente estejam atentos a essa problemática, e realizem uma orientação efetiva que venha a contribuir para a minimização dos erros de medicação no pós-alta hospitalar.

Quanto às dúvidas sobre o horário, tivemos a seguinte questão de 1 familiar:

Que horário ele vai tomar as medicações? (p. 32)
O enfermeiro anotou para cada medicamento da receita os horários em que o participante os utilizava durante a internação. Poderia nesse momento questionar ao participante sobre suas atividades diárias visando relacionar os horários de administração dos medicamentos as mesmas (uso antes ou após as refeições, antes de deitarse, etc.) respeitando as especificidades de cada medicamento.

Observamos assim que embora, no domicílio, algumas vezes os familiares sejam os responsáveis pela administração ao paciente, estes pouco têm sido orientados a esse respeito no momento da alta hospitalar do paciente. Verificamos ainda que mesmo quando questionam sobre o tratamento do paciente, recebem um número reduzido de informações.

O enfermeiro deve considerar que o paciente não é um ser abstrato e isolado, mas sim que encontra-se inserido em um contexto social e familiar e, desse modo, a mesma preocupação dispensada ao paciente deve ser dispensada à sua família, a qual deve ser compreendida como uma extensão do paciente ${ }^{(11)}$.

\section{CONCLUSÕES}

Os resultados dessa investigação permitiram concluir que:

- Os enfermeiros dispensaram um período curto de tempo para orientação ao participante $(92,1 \%$ realizaram a orientação em tempo inferior a 10 minutos) e desenvolveram essa atividade em locais inadequados (presença de ruídos, pessoas transitando, etc), o que pôde contribuir para distração do participante com conseqüente perda de informações relevantes à continuidade de seu tratamento;

- A maioria dos profissionais apenas leu a receita médica para o participante, sem identificar se o mesmo compreendeu as orientações fornecidas ou se, no domicílio, seria capaz de ler a receita caso esquecesse o modo de administração dos medicamentos;

- Orientações de enfermagem por escrito foram fornecidas apenas para $3(7,9 \%)$ participantes e limitaram-se às anotações quanto aos horários de administração dos medicamentos, na própria receita médica do participante;

- Onze participantes $(33,3 \%)$ apresentaram dúvidas durante a orientação final de enfermagem, sendo essas principalmente a respeito da dose e do nome dos medicamentos;
Administração de medicamentos: orientação final de enfermagem para a alta hospitalar 
Adriana Inocenti Miasso Silvia Helena De B. Cassiani
- Os participantes pouco questionaram a respeito da terapia medicamentosa instituída e, quando o fizeram, receberam um número reduzido de informações, sendo, essas, algumas vezes incorretas;

- Apesar de, em alguns casos, os familiares serem os únicos responsáveis pela administração dos medicamentos ao participante, esses pouco foram orientados a respeito da terapêutica medicamentosa instituída para o mesmo.

Assim, acreditamos que o reduzido número de orientações, a respeito da terapêutica medicamentosa, fornecidas ao participante e familiar durante a internação hospitalar, pode ter contri- buído para a ocorrência de erros de medicação no domicílio, após a alta hospitalar.

A partir dos dados encontrados nessa investigação sugerimos que:

- O enfermeiro, juntamente com a equipe de saúde, elabore um plano de alta individualizado para o paciente, a partir de sua admissão na instituição, com o objetivo de assegurar a continuidade de seu tratamento no domicílio;

- A equipe de saúde se mobilize em busca de novas estratégias de ensino, que atendam a necessidade individual do paciente, visando assegurar uma administração de medicamentos segura no domicílio.

\section{REFERÊNCIAS}

(1) Carvalho VT. Erros na administração de medicamentos: análise de relatos dos profissionais de enfermagem. [dissertação] Ribeirão Preto (SP): Escola de Enfermagem de Ribeirão Preto/USP; 2000.

(2) Mellone M. Hospital sem paredes: A desinternação é uma das metas para tornar o tratamento aos pacientes mais humanizado e baratear os custos gerais de internação hospitalar. Rev. Unimed Hosp Rec Próprios [periódico online] 1999. Disponível em: <http:/www.epub.com.br/unimed/hospital $>$. (10 mar. 1999)

(3) White MJ, Holloway M. Patient concerns after discharge from rehabilitation. Rehabil Nurs 1990; 15(6):316-8

(4) Zago MMF, Casagrande LDR. A comunicação do enfermeiro cirúrgico na orientação do paciente: a influência cultural. Rev Lat-Am Enferm 1997; 5(4):69-74.

(5) Aguillar OM. A alta do paciente cirúrgico no contexto do sistema de saúde. [dissertação] Ribeirão Preto (SP): Escola de Enfermagem de Ribeirão Preto/USP; 1990.

(6) Holloway A. Patient knowlwdge and information concerning medication on discharge from hospital. J Adv Nurs 1996; 24(6):1169-74.
(7) Lage OC. Preparo para alta pós-cirúrgica: resultados da ação andragógica observados durante a visita domiciliar. [dissertação] São Paulo (SP): Escola de Enfermagem da USP; 2002.

(8) Silva T, Dal-pizzol F, Bello CM, Mengue SS, Schenkel EP. Bulas de medicamentos e a informação adequada ao paciente. Rev Saúde Pública 2000; 34(2):184-9.

(9) Marin MJS. Levantamento de problemas dos pacientes idosos no momento da alta hospitalar. [dissertação] Ribeirão Preto (SP): Escola de Enfermagem de Ribeirão Preto/USP; 1995.

(10) Araújo RC. Aconselhamento ao paciente sobre medicamentos: ênfase nas populações geriátrica e pediátrica. [Apresentado no $3^{\circ}$ Encontro de Centros de Informação sobre Medicamentos do Brasil; 1999 out 27-30; Vitória].

(11) Andrade OG, Marcon SS, Silva DMP. Como os enfermeiros avaliam o cuidado/cuidador familiar. Rev Gaúcha Enferm 1997; 18(2):123-32.
Correspondência:

Adriana Inocenti Miasso

Rua José Urbano 170

BI. A2-Ap.46- Jd. Paulista

Ribeirão Preto

CEP -14091-190 - SP

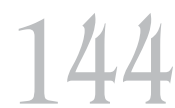

Rev Esc Enferm USP 2005; 39(2):136-44. 